\title{
VARIABILITY IN FORCE APPLIED TO THE HEAD DURING SIMULATED FACE MASK VENTILATION ON MANIKINS
}

\author{
C.R. Platten ${ }^{1}$, F.E. Wood ${ }^{2}$, S. Byrne ${ }^{2}$, I.R. Chambers ${ }^{1}$, J.P. Wyllie ${ }^{2}$ \\ ${ }^{1}$ Regional Medical Physics Department, Newcastle upon Tyne Hospitals NHS Foundation Trust, James Cook \\ University Hospital, ${ }^{2}$ Department of Neonatal Medicine, South Tees Hospitals NHS Trust, James Cook \\ University Hospital, Middlesbrough, UK
}

Background and aims: Excessive force applied to the head during delivery may cause injury and bradycardia. Force applied during mask positive pressure ventilation (PPV) has not previously been reported.

Applied force was observed to oscillate during PPV. We aimed to quantify the magnitude of this oscillation in relation to mask type and hold technique.

Methods: The force applied to a unique purpose built manikin (approx. 25/40,750g) during one minute of PPV was measured using a system designed to simultaneously capture this and respiratory data from a Florian monitor. Each of 72 participants, unaware force was being measured, used 6 different preterm masks from four manufacturers with a single handed ( $\mathrm{SH}$ ) and two handed (TH) (two-person technique) mask hold.

Force data was sampled and recorded at $200 \mathrm{~Hz}$ using Spectra ${ }^{\circledR}$ then analysed using LabChart ${ }^{\circledR}$ software. Pulse wave amplitude (difference between wave maxima and minima) was used as the measure of variability in applied force.

Results: Mean pulse wave amplitude differed significantly across mask type for SH $(\mathrm{p}<0.001)$ and TH mask holds $(\mathrm{p}<0.001)$.

Changing from SH to TH hold resulted in a statistically significant $50 \%$ reduction in both the mean $(0.41 \mathrm{~kg}$ to $0.21 \mathrm{~kg})$ and standard deviation $(0.37 \mathrm{~kg}$ to $0.18 \mathrm{~kg})$ of the pulse wave amplitude distributions $(\mathrm{p}<0.001)$.

Conclusions: Despite larger mean applied force, all masks showed reduced force variability with the TH compared to $\mathrm{SH}$ technique.

The physiological significance of the overall magnitude and variability in force applied to the neonatal head during resuscitation is unknown, but warrants further investigation. 